\title{
Hypoglycemia in the ICU: Discrepancy between capillary and serum glucose measurements in patients with renal disease
}

\author{
Ruth A. Verner, Maguy Chiha, Mary Ann Emanuele, Alaleh Mazhari \\ Division of Endocrinology and Metabolism, Department of Medicine, Loyola University Medical Center, Maywood, USA; \\ *Corresponding Author: amazhari@lumc.edu
}

Received 13 April 2013; revised 14 May 2013; accepted 10 June 2013

Copyright (C) 2013 Ruth A. Verner et al. This is an open access article distributed under the Creative Commons Attribution License, which permits unrestricted use, distribution, and reproduction in any medium, provided the original work is properly cited.

\begin{abstract}
Recently, a focus on tight glycemic control in intensive care units (ICU) has resulted in implementation of strict insulin protocols requiring frequent glucose monitoring. The use of pointof-care (POC) capillary glucose testing is widespread, but its validity in the ICU has been questioned. Our objective is to better understand the use of POC glucose at the extremes of glycemic control through a case review at our institution. We describe the case of a 75-year-old non-diabetic female with end stage renal disease (ESRD) on hemodialysis who was admitted with apparent hypoglycemia. After extensive workup was done for a seemingly refractive hypoglycemia, a discrepancy between POC capillary glucose and central serum glucose levels was discovered, revealing actual euglycemia and false low POC glucose values. Cases of hypoglycemia can be challenging, especially in non-diabetic patients with ESRD. While glucometers assessing capillary glucose are used both in the outpatient and inpatient environment, their validity in the critically ill patient has known limitations. Cases such as this have led to the development of systemic checks and balances, as well as further investigations regarding the use of $\mathrm{POC}$ glucose meters in the ICU. This case serves as a reminder to evaluate for all causes for abnormal laboratory values, including technological limitations.
\end{abstract}

Keywords: Point-of-Care; Hypoglycemia; ICU

\section{INTRODUCTION}

Patients with end stage renal disease (ESRD) on he- modialysis encounter a number of metabolic challenges, including difficulty with glycemic control [1]. Non-diabetic patients with renal failure are prone to hypoglycemia $[2,3]$, and at times, the etiology is difficult to determine. The differential diagnosis of hypoglycemia in the non-diabetic, non-critically ill patient with ESRD is broad including the following: exogenous sulfonylurea or insulin administration, adrenal insufficiency, non-islet cell tumors (tumor production of incompletely processed Insulin like Growth Factor IGF-1 or IGF-2), endogenous hyperinsulinism (beta cell tumors, insulinoma, functional beta cell disorder (nesidioblastosis)), insulin auto-immune hypoglycemia, critical illness, and malnourishment [1]. Additionally, because the kidney is vital for gluconeogenesis and glucose regulation, patients with chronic kidney disease often have compounding problems with glucose homeostasis and hypoglycemia $[3,4]$.

Patients at either extreme of glycemic control are often admitted to ICUs, where tight glycemic control is pursued. In the surgical ICU, strict glycemic control has been shown to improve morbidity and mortality [5]. However, intensive insulin therapy also carries the increased risk of hypoglycemia. In some patients, specifically those in medical intensive care units, this can be associated with worse outcomes [6,7]. Therefore, the reliability of glucose measurements in these clinical scenarios is essential in obtaining glycemic control while minimizing the risk of hypoglycemia.

Point-of-care (POC) glucose testing is commonly used both in outpatient and inpatient settings [8]. Numerous studies have investigated the reliability of POC glucose testing in critically ill patients. While the results in the normal hospital range show accuracy and reliability between POC testing and central laboratory testing, the results are less favorable in critical care settings and at extremes of glycemic control [9].

Overall, the complex clinical presentation, broad dif- 
ferential, and questionable reliability of glucose testing in critical care settings can make the care of a patient with ESRD on hemodialysis challenging.

In this case report, we describe a non-diabetic patient with ESRD on hemodialysis, without a history of glycemic control problems, who developed apparent persistent hypoglycemia as assessed by capillary glucose measurement. Eventually, the apparent hypoglycemia was attributed to discrepant POC and central glucose levels. We use this case as a reminder of the patient safety issues that can arise from the limitations of POC capillary glucose testing, especially in patients with ESRD and/or critical illness.

\section{CASE OF APPARENT HYPOGLYCEMIA IN THE ICU}

Our patient is a 75-year-old female with a history of end stage renal disease (ESRD) on hemodialysis (HD), hypertension (HTN), severe aortic stenosis, pulmonary HTN, hypothyroidism, anemia, sacral decubitus ulcers, morbid obesity. She had a rapid decline in health over the preceding six months, including dementia, episodes of sepsis, debilitating arthritis, and gallbladder surgery complicated by kidney failure for which she had become hemodialysis-dependent. On this occasion, she was admitted to the Medical ICU for apparent refractory hypoglycemia observed at a local nursing home. There, her reported blood sugar levels were $20 \mathrm{mg} / \mathrm{dl}-40 \mathrm{mg} / \mathrm{dl}$. Per family reports, she did not have a history of diabetes, but had experienced a similar episode of apparent hypoglycemia one month earlier, requiring hospital admission. No further details were available. The nursing home reported symptoms of increased fatigue and questionable mental status changes. However, in the emergency room (ER), she was unable to communicate with staff and was oriented to person only, making determination of changes from baseline difficult. As a result, laboratory testing was heavily relied upon for diagnoses and treatment.

Prior to arrival, she had received intravenous (IV) fluids containing dextrose $5 \%$ and $10 \%$. In the ER, initial fingerstick glucose revealed blood sugar of $127 \mathrm{mg} / \mathrm{dl}$, but subsequent sugars were repeatedly $<10 \mathrm{mg} / \mathrm{dl}$ on fingerstick and as a result, intramuscular glucagon was given. The timing of her last meal was not known. She was eventually started on a glucose drip and admitted to the ICU.

Initial endocrine workup showed low-normal random cortisol, with a normal cosyntropin stimulation test, and a mildly elevated thyroid stimulating hormone, making the diagnosis of panhypopituitarism unlikely. A growth hormone level was not checked. Sulfonylurea and repaglinide levels were negative, ruling out exogenous sulfonylurea or repaglinde administration. IGF-2 level was also normal. Computed Tomography of the pancreas was negative for pancreatic tumor and an octreotide scan was unrevealing for neuroendocrine tumors.

In the midst of the hypoglycemia workup, a discrepancy between glucose values from fingerstick-testing and central venous samples taken from her peripherally inserted central venous catheter (PICC) showed no evidence of significant hypoglycemia. Once this discrepancy was discovered, POC readings were taken only from PICC blood and were more closely concordant with serum glucose levels as measured on the basic metabolic panel.

Her later hospital course was complicated by septic shock from Proteus mirabilis bacteremia. After resolution of this episode, she re-developed a gram-negative rod bacteremia. She was eventually discharged without glycemic problems, but on antibiotics.

Figure 1 above shows the initial discrepancy between capillary glucose and serum glucose measurements in our patient. This discrepancy became less pronounced after approximately Day 8 when most POC glucose values were obtained from central blood.

\section{DISCUSSION}

This case presents multiple points of consideration for the care of critically ill patients, especially those with ESRD, who present with apparent hypoglycemia, especially in the inpatient setting. As this case dramatically illustrates, the validation of true hypoglycemia is crucial. If true hypoglycemia is present, as was the initial assumption in this case, it first reminds us of the wide differential for hypoglycemia, especially in light of the changes in glucose homeostasis that are common in patients with ESRD.

The kidney is vital for gluconeogenesis and glucose regulation. Thus, patients with ESRD are at risk for both hyper- and hypoglycemia. Both diabetics and non-dia-

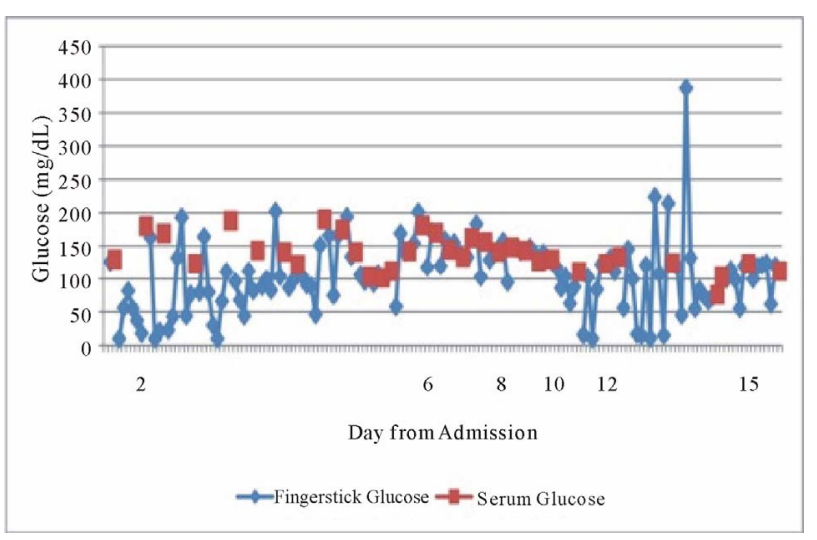

Figure 1. Longitudinal representation of serum glucose measurements (red) vs POC capillary (blue) glucose values in days from admission to hospital. 
betics can experience hypoglycemia, although their etiologies can be quite different. In patients with ESRD, hypoglycemia is more common than generally thought. The mechanisms of hypoglycemia in non-diabetics patients with ESRD include reduced clearance of insulin, sub-optimal gluconeogenesis in the kidneys and deficiencies of counter-regulatory hormones such as catecholamines and glucagon. In fact, renal gluconeogenesis plays a significant role in glucose homeostasis and should be considered along with the liver as an important site for gluconeogenesis [1,2].

In patients with diabetes, renal failure is also often accompanied by other complications of diabetes, and two of these make the blood glucose difficult to control. Gastroparesis makes it challenging to match food absorption and insulin delivery. Autonomic neuropathy may lead to hypoglycemia insensitivity. Anuric dialysis patients who do not have an osmotic diuresis have no escape valve and can have glucose levels $>1000 \mathrm{mg} / \mathrm{dl}$. These patients often appear better than other individuals with glucose levels this high because they are less volume contracted.

During hemodialysis, glucose is removed by filtration because it is a small molecule. The dialysis bath always contains glucose to prevent hypoglycemia. Thus if insulin is given at the start of dialysis, severe hypoglycemia can result. If not treated, the glucose will decrease, although not to target.

Second, and most poignantly, this case highlights a relatively common discrepancy that occurs between point-of-care capillary glucose readings and serum glucose measurements in the hospital setting. If not recognized, this discrepancy could result in serious health consequences as well as critical time and resource expenditures in the hospital setting.

It has been previously reported that significant discrepancies between capillary glucose measurements and central glucose values can exist [8-11]. Generally, it has been noted that no device has an error report of less than $5 \%$. Unfortunately inaccuracy rates can even exceed $>8.5 \%$ with some meters [10].

Capillary POC glucometer results must be interpreted in light of the patient's hematocrit level. Our patient's hematocrit ranged from 21.8 - 28.3 during her admission. At our institution, the glucometer manufacturer's (Accuchek) report states that hematocrit levels $<20 \%$ may yield falsely high glucose results and hematocrits $>55 \%$ may yield falsely low glucose results [12]. These differences are accounted for by the fact that red blood cells consume and contain significant amounts of glucose, thereby altering the whole blood levels depending on their concentration. Additionally, each meter technology will have different sensitivity to hematocrit [10]. To date, there is only one known meter that simultaneously measures hematocrit with glucose to offset this effect

\section{[11].}

Third, glucometers may measure other sugars, including maltose, galactose or xylose, commonly found in parental nutrition, which can falsely elevate glucose readings $[10,12]$.

Fourth, glucose measurement is a multistep process involving an enzymatic reaction and interference can occur at more than one step: the interaction of glucose with the enzyme, the transfer of electrons to the mediator from the enzyme and the interactions at the electrode. Substances that can falsely lower POC glucose results are extremely high triglycerides $(>4800)$ and elevated acetaminophen levels [10]. Substances that can falsely elevate POC glucose results are bilirubin $(>20 \mathrm{mg} / \mathrm{dL})$ and uric acid [10,12].

In addition to these rare cases, any medical condition that causes a decrease in blood flow to the extremity will make capillary glucose results unreliable. This is common in critical care situations where vasopressor agents cause peripheral vasoconstriction and restriction of blood flow to the extremities. Other similar situations include severe dehydration, DKA or non-ketotic hyperosmolar state, hypotension, shock or peripheral vascular disease.

Overall, studies have indicated that most glucose meters are relatively accurate in the outpatient setting, however this accuracy is not as clear-cut in the inpatient setting, especially among the critically ill [9]. Over the past decade, a renewed sense of focus on glycemic control in the critically ill has emerged since the landmark study by Van den Berghe in 2001. This study demonstrated that intensive insulin therapy improves outcomes in critically ill surgical patients with a target glucose of $80 \mathrm{mg} / \mathrm{dL}-110 \mathrm{mg} / \mathrm{dL}$. Subsequently, other studies failed to reproduce this finding. Specifically, results from the NICE SUGAR Study, in a multicenter setting, suggest that Van den Berghe's goal might be too rigorous, but that a glucose goal in the mid 100s was achievable and relatively safe [7]. On the other hand, it is known that certain patient populations, particularly cardiovascular patients, derive benefit from very strict glycemic control, as in Van den Berghe's protocol [6]. As a result of these studies, the consensus that closer attention to glycemic control in the critically ill has been widely accepted and hospitals across the country (especially in their ICUs) have adopted stricter insulin treatment protocols.

POC glucometers are suggested to have limitations in the ICU settings where many of these strict glycemic protocols are carried out. Even so, many ICU's use a combination of central venous glucose and POC glucose results to drive management of insulin protocols. While some studies continue to promote the use of certain POC glucometers in the intensive care setting [13], others continue to seriously question the use of POC glucometers in this setting $[9,14]$. As a result of this discrepancy, 
the Clinical and Laboratory Standards Institute (CLSI) is planning to release a new statement later this year clarifying the role of POC glucometers in the ICU. Until then, healthcare providers should be aware that POC accuracy in the intensive care setting continues to be debated.

In summary, our patient recovered to her baseline status and was discharged back to the nursing home. Even after the POC testing source was changed to the blood at her central access (PICC line), there were occasions when the POC glucose results were low and it is unclear if this was due to retesting a capillary sample, true hypoglycemia, or problems with the meter itself. Her case reminds us of the multiple sources of error inherent in POC testing and the caution that must be exercised in interpreting POC glucose values. Health care providers should critically interpret abnormal results and perform necessary checks in order to maintain patient safety.

Most institutions have protocols for glycemic management, therefore establishing a set of systemic checks and balances. With these in place, all healthcare providers are aware of the limitations of this technology, especially in critically ill patients. The protocols for continuous intravenous insulin infusion at our institution are as follows: after initiation of the insulin drip, fingerstick glucose is checked every hour until within goal range for 4 hours, and then fingerstick glucose levels are checked every 2 hours. Additionally, if patients are on pressors, require dialysis, or are hypotensive, thermally injured, or have severe hepatic dysfunction, severe peripheral edema, or requires more than 20 units/hour of insulin, serum blood glucose levels are drawn to confirm fingerstick reading twice daily. If at any time, there is a discrepancy of greater than $30 \mathrm{mg} / \mathrm{dL}$ between serum glucose and POC glucose, only the serum glucose measurements are used.

\section{CONCLUSION}

This case is a reminder to evaluate for all causes for abnormal laboratory values, including technological limitations. We suggest a precaution of drawing a serum or plasma sample for the central laboratory to confirm POC meter testing per our protocol above. We are not calling for the abandonment of POC glucometers in the inpatient hospital setting, but a renewed awareness of their limitations and a discerning mind when assessing results at the extremes of glycemia.

\section{REFERENCES}

[1] Cryer, P.E., et al. (2009) Evaluation and management of adult hypoglycemic disorders: An endocrine society cli- nical practice guideline. Journal of Clinical Endocrinology and Metabolism, 94, 709-728.

doi:10.1210/jc.2008-1410

[2] Mak, R.H.K. (2000) Impact of end-stage renal disease and dialysis on glycemic control. Seminars in Dialysis, 13, 4-8. doi:10.1046/j.1525-139x.2000.00007.x

[3] Strumvoll, M. (1998) Glucose production by the human kidney-Its importance has been underestimated. $\mathrm{Ne}$ phrology Dialysis Transplant, 13, 2996-2999. doi:10.1093/ndt/13.12.2996

[4] Triplett, C.L. (2012) Understanding the kidney's role in blood glucose regulation. American Journal of Managed Care, 18, S11-S16.

[5] Van den Berghe, G., et al. (2001) Intensive insulin therapy in critically ill patients. New England Journal of Medicine, 345, 1359-1367. doi:10.1056/NEJMoa011300

[6] Griesdale, D. et al. (2009) Intensive insulin therapy and mortality among critically ill patients: A meta-analysis including NICE-SUGAR study data. Canadian Medical Association Journal, 180, 821-827. doi:10.1503/cmaj.090206

[7] The Nice Sugar Investigators (2009) Intensive versus conventional glucose control in critically ill patients. The New England Journal of Medicine, 360, 1283-1297. doi:10.1056/NEJMoa0810625

[8] Khan, A.I., et al. (2006) The variability of results between point-of-care testing glucose meters and the central laboratory analyzer. Archives of Pathological Lab Medicine, 130, 1527-1532.

[9] Kanjii, S., et al. (2005) Reliability of point-of-care testing for glucose measurement in critically ill adults. Critical Care Medicine, 33, 2778-2785. doi:10.1097/01.CCM.0000189939.10881.60

[10] Ginsburg, B.H. (2009) Factors affecting blood glucose monitoring: Sources of error in measurement. Journal of Diabetes Science and Technology, 3, 903-913.

[11] Nichols, J.H. (2011) Blood glucose testing in the hospital: Error sources and risk management. Journal of Diabetes Science and Technology, 5, 173-177.

[12] Roche Diagnostics (2013) Hematocrit variable study protocol for glucose meter evaluations.

[13] Gijzen, K., et al. (2012) Is there a suitable point-of-care glucose meter for tight glycemic control? Evaluation of one home-use and four hospital-use meters in an intensive care unit. Clinical Chemistry and Laboratory Medicine, 50, 1985-1992.

[14] Critchell, C.D., et al. (2007) Accuracy of bedside capillary blood glucose measurements in critically ill patients. Intensive Care Medicine, 33, 2079-2084. 